\title{
Prevention of preeclampsia
}

\author{
Chad A. Grotegut
}

Division of Maternal-Fetal Medicine, Department of Obstetrics and Gynecology, Duke University, Durham, North Carolina, USA.

Preeclampsia is a common complication of pregnancy that is associated with an increased risk of maternal and neonatal morbidity and mortality. Currently, delivery is the only cure for preeclampsia; therefore, effective prevention and treatment options for this condition are sorely needed. In the current issue of the $J C l$, Mirzakhani et al. report the findings of the Vitamin D Antenatal Asthma Reduction Trial (VDAART), a well-conducted large, randomized, double-blind, placebo-controlled trial of vitamin D supplementation for the prevention of preeclampsia. Though vitamin D supplementation had no effect on the risk of preeclampsia, reduced maternal serum vitamin $D$ levels did correlate with preeclampsia risk. Mirzakhani and colleagues identified a number of gene pathways that are differentially regulated among women with low serum vitamin $D$ levels who develop preeclampsia. These results indicate that further research on the role of vitamin $D$ in preeclampsia is warranted.

\section{Preeclampsia and the burden of disease}

Preeclampsia is a disorder that is unique to human pregnancy, and the only known cure for this complication is delivery. Preeclampsia affects approximately $4 \%$ to $5 \%$ of pregnancies in the United States, is associated with significant maternal and neonatal morbidity, and is an important cause of maternal and neonatal mortality $(1,2)$. The etiology of preeclampsia is not known, but the placenta is an important mediator of the disease. Several lines of evidence have implicated abnormal remodeling of the spiral arterioles during early placental development and the resulting poor placental perfusion in the pathogenesis of preeclampsia (3). Interactions between uterine NK cells and invading trophoblasts may influence spiral arteriole remodeling, thereby demonstrating a potential immunologic mechanism for the development of the disorder (4). Hypoperfusion of the placenta leads to a relative hypoxic state within the placenta and the subsequent release of antiangiogenic peptides, such as soluble fms-like tyrosine kinase 1 (sFLT-1) and soluble endoglin (sENG), which antagonize VEGF and placental growth factor (PlGF) (5). These peptides lead to maternal endothelial damage that results in the preeclampsia-associated phenotypes of hypertension, proteinuria, and end-organ dysfunction (3).

Classic risk factors for preeclampsia include the extremes of maternal age (women in their early teens and those over the age of 40), nulliparity, new male partner, multiple gestations, obesity, chronic hypertension, preexisting diabetes, and other less-common medical conditions, such as renal disease, systemic lupus erythematosus, and antiphospholipid antibody syndrome. Despite this set of known risk factors, not all women with these risk factors develop the disorder, and not all women presenting with preeclampsia have a specific underlying risk factor (6) Because of the difficulty in predicting which women will develop this condition, much of the prenatal care in the United States, particularly in the third trimester, is directed at screening for preeclampsia

Related Article: p. 4702

Conflict of interest: The author has declared that no conflict of interest exists

Reference information: / Clin Invest. 2016;126(12):4396-4398. doi:10.1172/JCI91300.

(7). There is a great need to better identify those women at risk for preeclampsia and to develop effective preventative and therapeutic strategies for the disorder.

\section{Prevention of preeclampsia and the role of vitamin D}

As treatment and screening options are limited, therapies that prevent preeclampsia, delay the onset of the disorder, or decrease disease severity would be beneficial. One of the best-studied agents for the prevention of preeclampsia is lowdose aspirin. The US Preventive Services Task Force (USPSTF) and the American College of Obstetricians and Gynecologists (ACOG) currently recommend the use of low-dose aspirin for the prevention of preeclampsia in high-risk women (8). The number of at-risk women who need to be treated in order to prevent a single case of preeclampsia is based on the prevalence of the disorder. For example, in populations with a preeclampsia prevalence of $4 \%$ to $6 \%$, over 150 women need to be treated with low-dose aspirin to prevent a single case. However, despite initiation of this treatment, many women still present with early-onset, severe forms of the disease $(2,9)$. In addition to low-dose aspirin, other studied modalities for the prevention or treatment of preeclampsia include antioxidants (vitamins $\mathrm{C}$ and $\mathrm{E}$ ), calcium supplements, fish oil, nitric oxide supplements, nitric oxide donors, folic acid, weight loss, antihypertensive agents, and physical activity (2). Unfortunately, none of these modalities has shown much promise, though recent studies suggest that statin therapy may be potentially beneficial (10). While low serum levels of vitamin $\mathrm{D}$ have been associated with the development of preeclampsia, there are conflicting data as to whether vitamin D supplementation provides protection from the disorder (11-19).

In this issue, Mirzakhani et al. report the results of a planned secondary outcome from the Vitamin D Antenatal Asthma Reduction Trial (VDAART) (20). The VDAART was a randomized, double-blind, 
placebo-controlled clinical trial (clinicaltrials.gov NCT00920621) designed to evaluate vitamin D supplementation in addition to a daily multivitamin during pregnancy for the prevention of asthma or recurrent wheeze in offspring born to women or a biologic father with asthma, eczema, or allergic rhinitis (21). The group planned a secondary analysis to determine whether vitamin D supplementation also decreased the risk of preeclampsia (20). Mirzakhani and colleagues found that vitamin $\mathrm{D}$ supplementation during pregnancy increased serum vitamin D levels but did not affect the risk of preeclampsia. Despite this, women with sufficient serum 25-hydroxyvitamin D (25OHD) levels, defined as a serum level greater than or equal to $30 \mathrm{ng} / \mathrm{ml}$, during both early (1018 weeks) and late (32-38 weeks) pregnancy had a decreased risk of preeclampsia. The relationship between serum 25OHD levels and preeclampsia persisted, even when the data were controlled for potential confounding factors, including race/ ethnicity, BMI, parity, and location of the clinical site.

It is unclear why vitamin D supplementation in this trial, which raised serum 25OHD levels, did not influence the development of preeclampsia, as there was an observed dose response between serum $25 \mathrm{OHD}$ levels and the risk of preeclampsia. Women with higher serum 25OHD levels had an overall lower risk of preeclampsia, while women with lower serum 25OHD levels had an overall higher risk of preeclampsia. When analyzing the outcome data by randomization arm, it does not appear that Mirzakhani et al. stratified the results by baseline serum 25OHD status at the start of the trial, though these studies may have been underpowered to do so. It is possible that only women with low serum vitamin D levels at the start of pregnancy benefit from vitamin D supplementation, while women with serum vitamin D levels that are closer to the normal range do not benefit from supplementation. It is also possible that maternal vitamin D serum levels are a marker for some other factor that more directly affects placental function and risk for preeclampsia and that vitamin D supplementation would therefore not have an effect on the outcome. Finally, in the VDAART, all women, including the control subjects, received a multivitamin that contained 400 IU vitamin D. It is possible that this small amount of supplementation was enough to attenuate the risk for preeclampsia in the control group, leading to no observed effect on the outcome with supplementation.

The study also attempted to identify potential mechanisms that could help explain how vitamin D may influence the risk for preeclampsia. Mirzakhani et al. included an innovative nested case-control analysis among trial participants that determined differential peripheral blood gene expression profiles between women with and without preeclampsia by serum 25OHD status. A number of genes were identified that were differentially expressed among women with low serum $25 \mathrm{OHD}$ levels who went on to develop preeclampsia. These differentially regulated genes largely involved inflammatory and immune pathways, which, Mirzakhani and colleagues argue, provide a potential mechanism by which vitamin $\mathrm{D}$ affects preeclampsia. It is not clear how vitamin D may influence preeclampsia risk, but vitamin D likely plays a role in placental development, possibly by regulating genes associated with placental invasion and implantation as well as influencing immune reactions and inflammation at the maternal-fetal interface $(11,12,22,23)$.

\section{Study interpretation and future directions}

Multiple observational studies and randomized, controlled clinical trials have demonstrated an association of low serum vitamin $\mathrm{D}$ levels during pregnancy with an increased risk of preeclampsia (11-14, 17, 19). In addition, the findings of the VDAART reported by Mirzakhani et al. demonstrate a clear dose-response relationship between serum 25OHD levels and preeclampsia risk. The results of vitamin D supplementation trials with the goal of decreasing preeclampsia have largely been conflicting $(15,18,20)$. Variations in trial design, including the vitamin $\mathrm{D}$ dose regimens, the timing during pregnancy in which the intervention was started, and the baseline vitamin D status of the subjects may account for the different observed effects on preeclampsia. Furthermore, as outlined above, vitamin D status may be a marker for some other process that affects the risk for preeclampsia. Current- ly, ACOG does not recommend routine screening of vitamin D deficiency among all pregnant women, but does indicate that screening can be considered for women deemed high risk for vitamin D deficiency (24). For women with vitamin D deficiency, ACOG recommends supplementation with 1,000 to 2,000 IU per day but states that there is insufficient evidence at this time to recommend supplementation for the purpose of preventing preeclampsia (24). Given the results of the VDAART and other published trials on vitamin D supplementation in pregnancy, no changes in current guidelines for vitamin D screening or supplementation in pregnancy are warranted. And given the evidence that associates low serum vitamin D levels with hypertensive disorders of pregnancy, more intervention trials are clearly needed. It is possible that vitamin $\mathrm{D}$ supplementation in pregnancy may only benefit those women with low serum levels or may be beneficial when therapy is started prior to pregnancy. Well-designed randomized, controlled trials in which vitamin D supplementation is started prior to pregnancy or that focus only on women with low serum vitamin D levels may provide these answers. Effective interventions that decrease the incidence of preeclampsia can potentially decrease maternal and neonatal morbidity and mortality. Furthermore, preeclampsia has been linked to an increased risk for cardiovascular disorders later in life (25). It is possible that interventions that decrease the incidence of preeclampsia may also have the long-term benefit of decreasing cardiovascular complications later in life.

Address correspondence to: Chad A. Grotegut, Division of Maternal-Fetal Medicine, Duke University, DUMC Box 3967, Durham, North Carolina 27710, USA. Phone: 919.681.5220; E-mail: chad. grotegut@duke.edu.

1. HCUP Statistical Briefs Chronological. Healthcare Cost and Utilization Project (HCUP). Agency for Healthcare Research and Quality, Rockville, Maryland USA. HCUP Web site. www. hcup-us.ahrq.gov/reports/statbriefs/statbriefs. jsp. Accessed October 19, 2016.

2. American College of Obstetricians Gynecologists Task Force on Hypertension Pregnancy. Hypertension In Pregnancy. Washington, DC, USA: ACOG; 2013

3. Roberts JM, Hubel CA. The two stage model of preeclampsia: variations on the theme. Placenta. 
2009;30(Suppl A):S32-S37.

4. Moffett-King A. Natural killer cells and pregnancy. Nat Rev Immunol. 2002;2(9):656-663.

5. Levine RJ, et al. Circulating angiogenic factors and the risk of preeclampsia. $N$ Engl JMed. 2004;350(7):672-683.

6. North RA, et al. Clinical risk prediction for pre-eclampsia in nulliparous women: development of model in international prospective cohort. BMJ. 2011;342:d1875.

7. American Academy of Pediatrics American College of Obstetricians Gynecologists. Guidelines For Prenatal Care. 7th ed. Washington, DC, USA: AAP, ACOG; 2012.

8. LeFevre ML, US Preventive Services Task Force. Low-dose aspirin use for the prevention of morbidity and mortality from preeclampsia: U.S. Preventive Services Task Force recommendation statement. Ann Intern Med. 2014;161(11):819-826.

9. Askie LM, Duley L, Henderson-Smart DJ, Stewart LA, PARIS Collaborative Group. Antiplatelet agents for prevention of pre-eclampsia: a meta-analysis of individual patient data. Lancet. 2007;369(9575):1791-1798.

10. Costantine MM, Cleary K, Eunice Kennedy Shriver National Institute of Child Health Human Development Obstetric-Fetal Pharmacology Research Units Network. Pravastatin for the prevention of preeclampsia in high-risk pregnant women. Obstet Gynecol. 2013;121(2 pt 1):349-353.
11. Bodnar LM, Catov JM, Simhan HN, Holick MF, Powers RW, Roberts JM. Maternal vitamin D deficiency increases the risk of preeclampsia. JClin Endocrinol Metab. 2007;92(9):3517-3522.

12. Robinson CJ, Alanis MC, Wagner CL, Hollis BW, Johnson DD. Plasma 25-hydroxyvitamin D levels in early-onset severe preeclampsia. Am J Obstet Gynecol. 2010;203(4):366.e1-366.e6.

13. Shand AW, Nassar N, Von Dadelszen P, Innis SM, Green TJ. Maternal vitamin D status in pregnancy and adverse pregnancy outcomes in a group at high risk for pre-eclampsia. BJOG. 2010;117(13):1593-1598.

14. Xu L, Lee M, Jeyabalan A, Roberts JM. The relationship of hypovitaminosis $\mathrm{D}$ and IL-6 in preeclampsia. Am JObstet Gynecol. 2014;210(2):149.e1-149.e7.

15. Pérez-López FR, et al. Effect of vitamin D supplementation during pregnancy on maternal and neonatal outcomes: a systematic review and meta-analysis of randomized controlled trials. Fertil Steril. 2015;103(5):1278-1288.

16. De-Regil LM, Palacios C, Lombardo LK, Peña-Rosas JP. Vitamin D supplementation for women during pregnancy. Cochrane Database Syst Rev. 2016;(1):CD008873.

17. Tabesh M, Salehi-Abargouei A, Tabesh M, Esmaillzadeh A. Maternal vitamin D status and risk of pre-eclampsia: a systematic review and meta-analysis. J Clin Endocrinol Metab. 2013;98(8):3165-3173.
18. Haugen M, et al. Vitamin D supplementation and reduced risk of preeclampsia in nulliparous women. Epidemiology. 2009;20(5):720-726.

19. Hollis BW, Wagner CL. Vitamin D and pregnancy: skeletal effects, nonskeletal effects, and birth outcomes. Calcif Tissue Int. 2013;92(2):128-139.

20. Mirzakhani H, et al. Early pregnancy vitamin D status and risk of preeclampsia. JClin Invest. 2016;126(12):4702-4715.

21. Litonjua AA, et al. Effect of prenatal supplementation with vitamin $\mathrm{D}$ on asthma or recurrent wheezing in offspring by age 3 years: The VDAART Randomized Clinical Trial. JAMA. 2016;315(4):362-370.

22. Fischer D, et al. Metabolism of vitamin D3 in the placental tissue of normal and preeclampsia complicated pregnancies and premature births. Clin Exp Obstet Gynecol. 2007;34(2):80-84.

23. Makris A, Xu B, Yu B, Thornton C, Hennessy A. Placental deficiency of interleukin-10 (IL-10) in preeclampsia and its relationship to an IL10 promoter polymorphism. Placenta. 2006 ; $27(4-5): 445-451$.

24. ACOG Committe on Obstetric Practice. ACOG Committee Opinion No. 495: Vitamin D: screening and supplementation during pregnancy. Obstet Gynecol. 2011;118(1):197-198.

25. McDonald SD, Malinowski A, Zhou Q, Yusuf S, Devereaux PJ. Cardiovascular sequelae of preeclampsia/eclampsia: a systematic review and meta-analyses. Am Heart J. 2008;156(5):918-930. 